\title{
Transverse Momentum Distribution of Atoms in an Interferometer
}

\author{
Mirjana Božić, Dušan Arsenović, and Lepša Vuškovića \\ Institute of Physics, P.O. Box 57, 11000 Beograd, Yugoslavia \\ a Old Dominion University, Department of Physics, \\ 4600 Elkhorn Avenue, Norfolk, VA 23529, USA
}

Reprint requests to Dr. D. A.; Fax: 381-11-3162190, E-mail: arsenovic@phy.bg.ac.yu

Z. Naturforsch. 56 a, 173-177 (2001); received February 8, 2001

\begin{abstract}
Presented at the 3rd Workshop on Mysteries, Puzzles and Paradoxes in Quantum Mechanics, Gargnano, Italy, September 17 - 23, 2000.
\end{abstract}

\begin{abstract}
From the stationary solution of Schrödinger's equation in an interferometer we derive the wave functions of the longitudinal and the transverse motion. The former function is a plane wave. The wave function of the transverse motion is a one dimensional wave packet representing a superposition of components with various values of the particles transverse momentum. The particles transverse momentum distribution in an interferometer is time independent and is determined by the aperture wave function. Consequently, it is independent of the distance from the slits. As such, it is a very important characteristic of the quantum state. Experimental determination of the momentum distribution would support the particle and wave interpretation of quantum interference in a new manner.
\end{abstract}

Key words: Atomic Interference; Transfer Momentum Distribution; Wave-particle Duality.

\section{Introduction}

Double and multiple slit experiments with massive particles, like the neutron experiment of Zeilinger $e t$ al. [1], the electron experiment of Tonomura et al. [2], the sodium atom experiment of Keith et al. [3], the helium atom experiments of Carnal and Mlynek [4] and of Kurtsiefer et al. [5], the $\mathrm{C}_{60}$ molecule experiment of Arndt et al. [6] and the similar experiment with photons [7] clearly show that electrons, neutrons, atoms, molecules and photons act as both waves and particles [8]. But, the clear understanding of the relation between wave and particle properties is still lacking [9].

Classical interference experiments were interpreted as demonstrating wave properties of quantons. In the new generation of experiments, in addition to the wave aspect, the particle aspect has been demonstrated, too. Time evolution of the interference pattern [2], or the time of arrival of particles to the detector $[4,10]$, are essential features of the new interference experiments. Thus, it is needed to determine and to study the nonstationary solutions $\Psi(x, y, t)$ of the Schrödinger equation in an interferometer

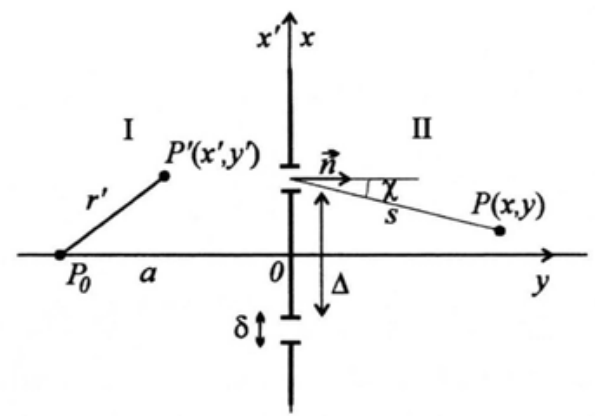

Fig. 1. Illustration of the geometry for the Fresnel-Kirchhoff diffraction formula (4).

$$
-\frac{\hbar^{2}}{2 m}\left(\frac{\partial^{2}}{\partial x^{2}}+\frac{\partial^{2}}{\partial x^{2}}\right) \Psi(x, y, t)=i \hbar \frac{\partial}{\partial t} \Psi(x, y, t),(1)
$$

where the initial state is a wave packet which describes particles arriving at the slits. These solutions have to satisfy the appropriate boundary conditions at the border between regions I and II (Figure 1).

In the search for a general time dependent solution of the two-dimensional problem, as an intermediate step, we determine the nonstationary solution of the 
(one-dimensional) transverse motion behind the slits, $\psi_{\mathrm{tr}}(x, t)$. The longitudinal motion in front and behind the slits we describe by a plane wave $e^{i p y / \hbar}$, where $\vec{v}=v \vec{i}_{y}$ is the initial particle longitudinal velocity and $p=m v$.

\section{Time Dependent Wave Function of the Transverse Motion}

Let us denote the stationary solution of (1) by $\Psi(x, y, t)=e^{i \omega t} \varphi(x, y)$, where $\varphi(x, y)$ satisfies the Helmholtz equation

$$
-\frac{\hbar^{2}}{2 m}\left(\frac{\partial^{2}}{\partial x^{2}}+\frac{\partial^{2}}{\partial y^{2}}\right) \varphi(x, y)=\hbar \omega \varphi(x, y),
$$

and the appropriate boundary conditions. We shall derive the function $\psi_{\operatorname{tr}}(x, t)$ from $\varphi(x, y)$ by generalizing the method apllied in [5] to determine $\left|\psi_{\text {tr }}(x, t)\right|$ from $|\varphi(x, y=v t)|$, where $\hbar \omega=m v^{2} / 2$. The method is based on the definition

$$
\left|\psi_{\mathrm{tr}}(x, t)\right|^{2} \equiv \frac{\left|\varphi_{\mathrm{FK}}(x, y=v t)\right|^{2}}{\int \mathrm{d} x\left|\varphi_{\mathrm{FK}}(x, y=v t)\right|^{2}}
$$

where $\varphi_{\mathrm{FK}}(x, y)$ is the solution of (1) in the FresnelKirchhoff form [11, 12]

$$
\begin{gathered}
\varphi_{\mathrm{FK}}(x, y)=A \frac{e^{i k r^{\prime}}}{r^{\prime}}, y \leq 0, \\
\varphi_{\mathrm{FK}}(x, y)=-\frac{i A}{2 \lambda} \frac{e^{i k a}}{a} \int_{\mathcal{A}} \mathrm{d} x^{\prime} \frac{e^{i k s}}{s}[1+\cos \chi], y \geq 0 .
\end{gathered}
$$

Here $s=\sqrt{y^{2}+\left(x^{\prime}-x\right)^{2}}, \cos \chi=y / s, \lambda=2 \pi / k=$ $h / p, A$ is a constant while $\mathcal{A}=\left\{x^{\prime} ;-(\Delta / 2)-\delta<\right.$ $\left.x^{\prime}<-(\Delta / 2)\right\}$ when one slit is open and other is closed, and $\mathcal{A}=\left\{x^{\prime} ;(\Delta / 2)<x^{\prime}<(\Delta / 2)+\delta\right.$ or $\left.-(\Delta / 2)-\delta<x^{\prime}<-(\Delta / 2)\right\}$ when the two slits are open. It is assumed that the distance $a$ of the doubleslit screen from the source $P_{0}$ is very large compared to the width of the slits (see Figure 1).

The function $\left|\psi_{\mathrm{t} r}(x, t)\right|^{2}$ defined by (3) is presented graphically in Figs. 2 and 3 for one and two open slits, respectively. In Figs. 2 and 3 one clearly sees the Fresnel (near field) region and the Fraunhofer (far field) region. The interferometer parameters are chosen from the atom interference experiment of $[5,10]$. It is important to point out that in this work the relation

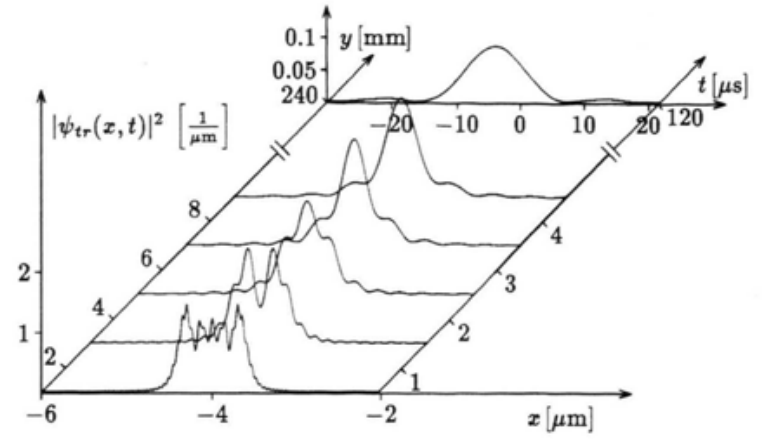

Fig. 2. The function $\left|\psi_{\mathrm{rr}}(x, t)\right|^{2}$ for a particle of mass $m=$ $6.6432 \cdot 10^{-27} \mathrm{~kg}$ (He atom) behind the single slit $(\delta=$ $1 \mu \mathrm{m})$. The function was evaluated from (3) and from (9), where $c\left(p_{x}\right)$ is given in (12a). The curves obtained in these two ways are indistinguishable. For the evaluation from (3) it is necessary to specify also the longitudinal velocity $v$ (or the de Broglies wave vector $k$ ). We choosed the value $k=4 \pi \cdot 10^{10} \mathrm{~m}^{-1}(v=\hbar k / m=1995.58 \mathrm{~m} / \mathrm{s})$. This value is unnecessary for the evaluation from (9), but the value of $v$ is necessary to establish the correspondance between the $t$ - and $y$ axis.

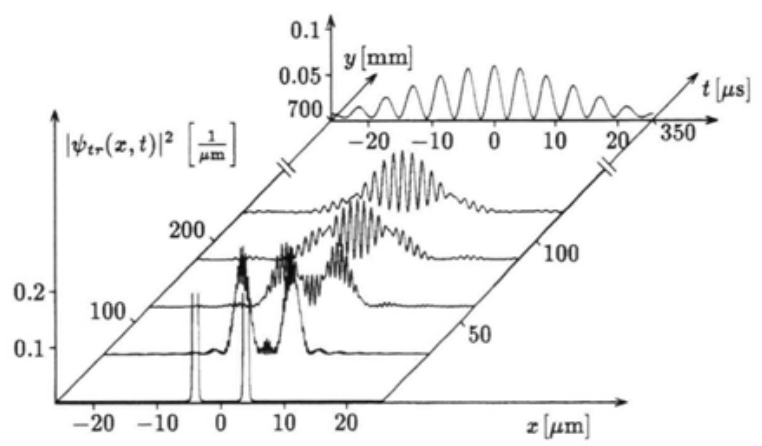

Fig. 3. The function $\left|\psi_{\mathrm{rr}}(x, t)\right|^{2}$ behind the double slit $(\delta=$ $1 \mu \mathrm{m}, \Delta=7 \mu \mathrm{m}$ ) evaluated from (3) and from (9), where $c\left(p_{x}\right)$ is given in (12b). The curves obtained in these two ways are indistinguishable. The parameters are the same as in Figure 2.

$y=v t$ is essential. First, it was used in (3). Second, it was used in the measurement of $\left|\psi_{\text {tr }}(x, t)\right|^{2}$, which is independent of $v$. Due to the large spread of atomic initial velocities, their time of arrival $(t=y / v)$ to a point $(x, y)$ takes different values in a large interval. By measuring the atomic time of arrival at various points on the line parallel to slits, at the fixed distance $y$, the authors were able to measure $\left|\psi_{\mathrm{tr}}(x, t)\right|^{2}$.

Our method of deriving $\psi_{\mathrm{tr}}(x, t)$ from $\varphi(x, y)$ is based on a different form of the solution of the Helmholtz equation. This form is a linear superpo- 
sition of eigenfunctions $e^{i p_{x} x / \hbar}$ and $e^{i \sqrt{p^{2}-p_{x}^{2}} \cdot y / \hbar}$ of momenta $\hat{p}_{x}$ and $\hat{p}_{y}$, respectively. It is straightforward to verify that the function

$$
\begin{gathered}
\varphi(x, y)=A e^{i p y / \hbar}, y \leq 0 \\
\varphi(x, y)=\frac{1}{\sqrt{2 \pi \hbar}} \int_{-\infty}^{+\infty} \mathrm{d} p_{x} c\left(p_{x}\right) e^{i p_{x} x / \hbar} e^{i \sqrt{p^{2}-p_{x}^{2}} \cdot y / \hbar} \\
y \geq 0
\end{gathered}
$$

satisfies the Helmholtz equation. Here $p=m v$, $p^{2} / 2 m=\left[p_{x}^{2}+p_{y}^{2}\right] / 2 m=\hbar \omega, A$ is a constant and $c\left(p_{x}\right)$ is an arbitrary function. The arbitrariness of $c\left(p_{x}\right)$ is removed, after imposing the normalization condition $\int_{-\infty}^{+\infty} \mathrm{d} p_{x}\left|c\left(p_{x}\right)\right|^{2}=1$ and the boundary condition

$\varphi(x, 0)= \begin{cases}1 / \sqrt{\delta}, & -(\Delta / 2) \geq x \geq-(\Delta / 2)-\delta, \\ 0, & \text { all other values of } x\end{cases}$

for one open slit, and

$\varphi(x, 0)=\left\{\begin{array}{l}1 / \sqrt{2 \delta},-(\Delta / 2) \geq x \geq-(\Delta / 2)-\delta, \\ 1 / \sqrt{2 \delta},(\Delta / 2)+\delta \geq x \geq(\Delta / 2), \quad \text { all other values of } x \\ 0, \quad 6\end{array}\right.$

for two open slits.

Let us assume that $c\left(p_{x}\right)$ tends to zero when $p_{x} / p \ll 1$ is violated. Using the approximation $\sqrt{p^{2}-p_{x}^{2}} \approx p-\frac{p_{x}^{2}}{2 p}$, the function $\varphi(x, y)$ in (5) is easily transformed to the form

$$
\begin{array}{r}
\varphi(x, y)=\frac{1}{\sqrt{2 \pi \hbar}} e^{i p y / \hbar} \int_{-\infty}^{+\infty} \mathrm{d} p_{x} c\left(p_{x}\right) e^{i p_{x} x / \hbar} \\
\cdot e^{-i p_{x}^{2} y / 2 p \hbar}, y \geq 0,
\end{array}
$$

By substituting the latter form into $\Psi(x, y, t)=$ $e^{i \omega t} \varphi(x, y)$ we obtain

$$
\begin{aligned}
\Psi(x, y, t) & =\frac{1}{\sqrt{2 \pi \hbar}} e^{-i \omega t} e^{i p y / \hbar} \\
& \cdot \int_{-\infty}^{+\infty} \mathrm{d} p_{x} c\left(p_{x}\right) e^{i p_{x} x / \hbar} e^{-i p_{x}^{2} y / 2 p \hbar}, y \geq 0 .
\end{aligned}
$$

The first two terms represent a plane wave which propagates along the $y$ axis. The second term (the integral) depends on $y$ and $x$. Now we invoke wave particly duality and start to think about a particle surrounded by the wave of (8). Its form suggests that a particle arriving to the slits continues to move with longitudinal momentum $p=m v$ along $y$-axis. But there is a probability density $c\left(p_{x}\right)$ that it aquires a value $p_{x}$ of the transverse momentum. So, its motion (evolution) along the $x$ axis is nonstationary and it is described by a nonstationary solution of the Schrödinger equation. We derive the wave function $\psi_{\mathrm{tr}}(x, t)$ from (7) by substituting the value $y=v t$ of the particle's $y$-coordinate at time $t$ in the integral. So,

$$
\begin{array}{r}
\psi_{\mathrm{tr}}(x, t)=\frac{1}{\sqrt{2 \pi \hbar}} \int_{-\infty}^{+\infty} \mathrm{d} p_{x} c\left(p_{x}\right) e^{i p_{x} x / \hbar} e^{-i \omega_{x} t}, \\
y=v t, y \geq 0,
\end{array}
$$

where $\omega_{x} \equiv p_{x}^{2} / 2 m \hbar$ naturally arises from $p_{x}^{2} y / 2 p \hbar=$ $p_{x}^{2} v t / 2 p \hbar=p_{x}^{2} t / 2 \hbar m$.

We see that $\psi_{\mathrm{tr}}(x, t)$ has the form of the general solution of the one-dimensional Schrödinger equation for a free quanton. The functions $\varphi(x, y=v t)$ and $\psi_{\mathrm{tr}}(x, t)$ satisfy the (3), used in $[5,10]$ to determine $\left|\psi_{\mathrm{tr}}(x, t)\right|^{2}$ from $|\varphi(x, y=v t)|^{2}$. The method used here to determine $\psi_{t r}(x, t)$ from $\varphi(x, y)$ is a generalization of the method $[5,10]$ based on the FresnelKirchhoff integral. This is because the wave function itself has been determined here, while in $[5,10]$ only its modulus square was determined.

By evaluating the function $\left|\psi_{\mathrm{tr}}(x, t)\right|^{2}$ given in (9), identical graphs are obtained with the graphs obtained from the Fresnel-Kirchhoff integral and presented in Figs. 2 and 3. From this fact we conclude that the function $\left|\psi_{\mathrm{tr}}(x, t)\right|^{2}$ defined in (9) and the function $\left|\varphi_{\mathrm{FK}}(x, y=v t)\right|^{2} / \int \mathrm{d} x\left|\varphi_{\mathrm{FK}}(x, y=v t)\right|^{2}$ are identical. However, we have not yet found a general proof of that fact.

\section{The Wave Function of the Transverse Motion in the Momentum Representation}

The function $c\left(p_{x}\right)$ in the state $\psi_{\mathrm{tr}}(x, t)$ is determined by the boundary condition of (6):

$$
\varphi(x, 0)=\frac{1}{\sqrt{2 \pi \hbar}} \int_{-\infty}^{+\infty} \mathrm{d} p_{x} c\left(p_{x}\right) e^{i p_{x} x / \hbar} .
$$

From the latter condition follows

$$
c\left(p_{x}\right)=\frac{1}{\sqrt{2 \pi \hbar}} \int_{-\infty}^{+\infty} \mathrm{d} x \varphi(x, 0) e^{-i p_{x} x / \hbar} .
$$



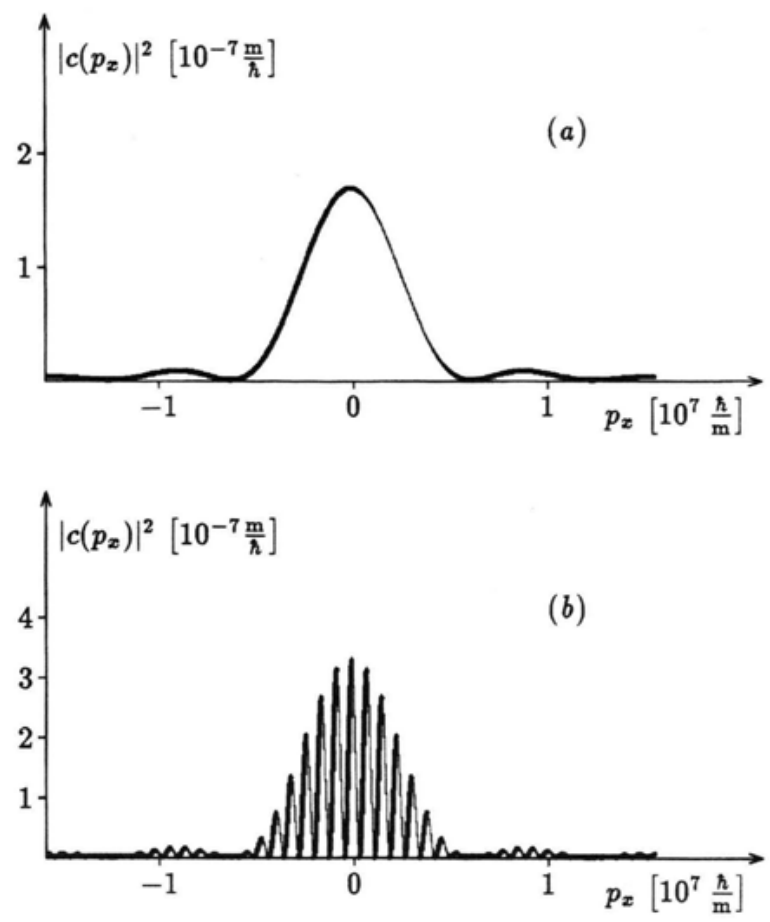

Fig. 4. The particles transverse momentum distributions $\left|c\left(p_{x}\right)\right|^{2}$ behind the (a) single slit and (b) double slit. They are evaluated by performing the Fourier transform of $\varphi_{\mathrm{FK}}(x, y)$ (for various fixed values of $y$ ) and from (12a) and (12b), respectively. The curves obtained from the two methods are indistinguishable. The parameters are the same as in Figure 2.

By substituting the functions $\varphi(x, 0)$ given in (6), one finds

$$
c\left(p_{x}\right)=\sqrt{2 \hbar} \frac{e^{i p_{x}(\Delta+\delta) / 2 \hbar}}{p_{x} \sqrt{\pi \delta}} \sin \left(p_{x} \delta / 2 \hbar\right)
$$

for one open slit and

$c\left(p_{x}\right)=\frac{2 \sqrt{\hbar}}{p_{x} \sqrt{\pi \delta}} \sin \left(p_{x} \delta / 2 \hbar\right) \cdot \cos p_{x}(\Delta+\delta) / 2 \hbar$

for two open slits. We see that $c\left(p_{x}\right)$ is independent of the initial longitudinal momentum and independent of the coordinate $y$. Consequently, the wave function $\psi_{t r}(x, t)$ of the transverse motion is also independent of the initial longitudinal momentum. We want to point out that $\psi_{t r}(x, t)$ is determined assuming that $c\left(p_{x}\right)$ is negligible when $p_{x} \ll p$ is violated.
The function $C\left(p_{x}, t\right) \equiv c\left(p_{x}\right) e^{-i \omega_{x} t}$ is a wave function of the transverse motion in the momentum representation. Its modulus square $\left|C\left(p_{x}, t\right)\right|^{2}=$ $\left|c\left(p_{x}\right)\right|^{2}$ is a probability density of the particles transverse momentum in an interferometer. The momentum distributions $\left|c\left(p_{x}\right)\right|^{2}$ for one and two open slits are given in Figure 4. By comparing these two momentum distributions, one concludes that the presence of the second slit influences the transverse momentum of each atom, independent of the slit through which it has passed to region II. Certain values of the particle's transverse momentum, which are allowed with one slit, are not allowed when both slits are open.

\section{Conclusion - Compatibility of Wave and Particle Properties}

One of the key statements in [12] is: the field distribution in the Fraunhofer diffraction pattern is the Fourier transform of the field distribution across the aperture (i.e. the aperture function). The statement follows from the Fresnel-Kirchhoff solution (4) of the Helmholtz equation using the approximation $s \approx y-x x^{\prime} / y$, which is valid very far from the slits $[11,12]$. Then, the function $\varphi_{\mathrm{FK}}(x, y)$ takes the form

$\varphi_{F K}(x, y) \approx-\frac{i A}{\lambda} \frac{e^{i k a}}{a} \frac{e^{i k y}}{y} \int_{\mathcal{A}} \mathrm{d} x^{\prime} e^{-i k x x^{\prime} / y}$,

$y$ very large.

By introducing the apperture function, which is proportional to $\varphi\left(x^{\prime}, 0\right)$, the latter equation is generalized to the equation

$$
\varphi(x, y) \approx \frac{D(y)}{\sqrt{2 \pi}} \int_{-\infty}^{+\infty} \mathrm{d} x^{\prime} \varphi\left(x^{\prime}, 0\right) e^{-i k x x^{\prime} / y},
$$

$y$ very large,

where $D(y)$ is a function of $y$. Based on this expression, $\mathrm{d} \varphi(x, y)$ at a given point $P$ with coordinate $x, y$ is envisioned as if it were a plane wave propagating in the direction connecting a point $P$ with the origin $O$ (Fig. 1) and having an amplitude determined by $\varphi\left(x^{\prime}, 0\right) \mathrm{d} x^{\prime}$. For each point on the image line, there is a corresponding spatial frequency (component of a wave vector) $K_{x} \equiv k x / y$ [12] so that 


$$
\begin{aligned}
\varphi(x, y) & =\frac{D(y)}{\sqrt{2 \pi}} \int_{-\infty}^{+\infty} \mathrm{d} x^{\prime} \varphi\left(x^{\prime}, y=0\right) e^{-i K_{x} x^{\prime}} \\
& =D(y) \mathcal{F}\left(K_{x}\right), y \text { very large. }
\end{aligned}
$$

Therefore, very far from the slits the field distribution $\varphi(x, y)$ is proportional to the Fourier transform $\mathcal{F}$ of the wave field accross the aperture.

By comparing (11) and (15) we see that the functions $c\left(p_{x}\right)$ and $\mathcal{F}\left(K_{x}\right)$ are identical up to a proportionality constant. Their arguments $p_{x}$ and $K_{x}$ respectively, have different physical meaning. This difference reflects the difference of the physical picture behind the functions $c\left(p_{x}\right)$ and $\mathcal{F}\left(K_{x}\right)$. The function $c\left(p_{x}\right)$ is a function of a particle's momentum. Its modulus square $\left|c\left(p_{x}\right)\right|^{2}=\left|C\left(p_{x}, t\right)\right|^{2}$ is a probability density of the particles transverse momentum in an interferometer. $\mathcal{F}\left(K_{x}\right)$ is a function of a wave vector $K_{x} \equiv k x / y . \mathcal{F}\left(K_{x}\right)$ expresses the wave character of the quantum wave function and $c\left(p_{x}\right)$ expresses the particle character of a wave function. It is a very important characteristic of the quantum state which does not depend on the $y$-coordinate that is the distance from the slit.

[1] A. Zeilinger, R. Gähler, C. G. Schull, W. Treimer, and W. Mampe, Rev. Mod. Phys. 60, 1067 (1988).

[2] A. Tonomura, J. Endo, J. Matsuda, T. Kawasaki, and H. Ezawa, Amer. J. Phys. 57, 117 (1989).

[3] W. Keith, C. R. Ekstrom, Q. A. Turchette, and D. E. Pritchard, Phys. Rev. Lett. 66, 2693 (1991).

[4] O. Carnal, and J. Mlynek, Phys. Rev. Lett. 66, 2689 (1991).

[5] Ch. Kurtsiefer, T. Pfau, and J. Mlynek, Nature (London) 386, 150 (1997).

[6] M. Arndt, G. Nairz, J. V. Andreae, C. Keller, G. van der Zouw, and A. Zeilinger, Nature (London) 401, 681 (1999).

[7] P. Grangier, G. Roger, and A. Aspect. Europhys. Lett. 1, 173 (1986).
This dual character is seen in $\psi_{\text {tr }}(x, t)=\varphi(x$, $y=v t$ ), too. From one side, it is a superposition of waves (see (15)) spreading from the slits points. From the other side, its modulus square $\left|\psi_{\mathrm{tr}}(x, t)\right|^{2}=$ $|\varphi(x, y=v t)|^{2}$ is a probability density of the particles position at the point $(x, y)$ and time $t=y / v$.

We would conclude from all these facts that both characters are simultaneously present in an interferometer. The two meanings of the Fourier transform of the aperture function support in a new way the de Broglie understanding of the wave particle duality and of the relation $\hbar k=p$. According to this understanding, wave and particle properties are compatible and simultaneously present.

To the best of our knowledge, the quanton transverse momentum distribution $\left|c\left(p_{x}\right)\right|^{2}$ in a single slit and a double slit interferometer has not been measured. However, we pointed out here and in [13] that it is a very important characteristic of the quantum state. Experimental evidence of the transverse momentum distribution would support the particle and wave interpretation of quantum interference in a new manner.

[8] S. T. Thornton and A. Rex, Modern Physics for scientists and engineers (Sound. Coll. Pub., Fort Worth, 1993).

[9] H. Kaiser, R. Clothier, S. A. Werner, H. Rauch, and H. Wolwitsch, Phys. Rev. A45, 31 (1992).

[10] T. Pfau and Ch. Kurtsiefer, J. Mod. Opt. 44, 2551 (1997).

[11] M. Born and E. Wolf, Principles of Optics, Pergamon Press, Oxford 1965.

[12] E. Hecht, Opticsm, Addison-Wesley, Reading, MA 1987.

[13] L. Vušković, D. Arsenović, and M. Božić, submitted to Phys. Lett. A. 